seine Zuversicht verstärkt, dass die Sache des Friedens und des Sozialismus den Sieg davontragen werde.

Das Gespräch dauerte 1 Stunde und 45 Minuten

Protokolliert von D. P. Schewljagin

Übersetzt aus dem russischen Originaltext

RGANI, fond 52, opis' 1 , delo 568, Bl.3-11

\title{
3. Stellungnahme Chruschtschows bei Eröffnung der Tagung der kommunistischen und Arbeiterparteien der sozialistischen Länder am 6. Januar 1956
}

Chruschtschow: Genossen! Ich denke, wir können mit der Arbeit beginnen. Gestattet mir einige Worte.

Im zurückliegenden Jahr 1955 haben die Sowjetunion, die Chinesische Volksrepublik, Polen, die Tschechoslowakei, Ungarn, die Deutsche Demokratische Republik, Rumänien, Bulgarien, Albanien und die anderen Länder des sozialistischen Lagers gewaltige Erfolge im Kampf um die Konsolidierung ihrer außenpolitischen Positionen und die Festigung des Friedens erzielt. In dieser Zeit hat sich die brüderliche Zusammenarbeit zwischen unseren Ländern weiter verstärkt.

Das sozialistische Lager ist zu einem wahren Bollwerk aller fortschrittlichen Kräfte der Welt geworden. Die Wirtschaft jedes der Staaten, die zu diesem Lager gehören, hat sich deutlich entwickelt und konsolidiert.

$\mathrm{Zu}$ den wichtigsten Errungenschaften des sozialistischen Lagers sind vor allem solche Ereignisse zu zählen wie der Friedensvertrag mit Österreich, ${ }^{35}$ die Beilegung des Konflikts mit Jugoslawien, ${ }^{36}$ die Aufnahme diplomatischer Beziehungen zwischen der UdSSR und der Deutschen Bundesrepublik, ${ }^{37}$ die Festigung der Beziehungen zwischen der UdSSR und Finnland dank der vorzeitigen Rückgabe des Militärstützpunkts Porkkala-Udd an die Finnen, ${ }^{38}$ die Reduzierung der Streitkräfte der Sowjetunion und Länder der Volksdemokratien. ${ }^{39}$ Eine große positive Rolle spielten auch die Besuche parlamentarischer Delegationen aus vielen

35 Am 15. Mai 1955 wurde der Staatsvertrag zwischen den vier Hauptsiegermächten des Zweiten Weltkriegs und Österreich geschlossen, der zwar die Funktion eines Friedensvertrags hatte, aber nicht als solcher bezeichnet wurde, weil Österreich nach amtlicher Lesart nicht zu den Kriegsgegnern gehört hatte, sondern als befreites Land anzusehen war.

36 Vom 26. Mai bis 2. Juni 1955 waren Chruščëv und Bulganin zur Wiederherstellung der 1948 abgebrochenen Parteibeziehungen in Belgrad.

37 In Verhandlungen vom 9. bis 13. September 1955 vereinbarte die sowjetische Führung mit Bundeskanzler Adenauer die Aufnahme diplomatischer Beziehungen.

38 Bei der Erneuerung des Beistandsvertrags mit Finnland am 19. September 1955 war die UdSSR zur Aufgabe des an die finnische Hauptstadt angrenzenden Marinestützpunkts Porkkala-Udd bereit, den sie gemäß dem Friedensvertrag von 1947 für 50 Jahre gepachtet hatte.

39 Die UdSSR fasste 1955 zwei Beschlüsse über die Reduzierung ihres Militärpersonals um insgesamt 640000 Mann. Im Zusammenhang damit konnten auch die mit ihr verbündeten Staaten ihre Truppenzahl vermindern. 
Ländern in der Sowjetunion, die Reise Nehrus in die UdSSR,40 die Einberufung der Bandung-Konferenz, auf der die Chinesische Volksrepublik eine aktive Rolle gespielt hat, ${ }^{41}$ [und] die Aufnahme von 16 neuen Staaten in die Vereinten Nationen, darunter Albanien, Bulgarien, Ungarn und Rumänien.

Ein großes internationales Ereignis war die Konferenz der Regierungschefs der vier Mächte in Genf. ${ }^{42}$ Die regierenden Kreise der Vereinigten Staaten von Amerika, Englands und Frankreichs waren dazu genötigt, auf diese Konferenz einzugehen. Sie sind zu dieser Konferenz gekommen unter dem Einfluss der öffentlichen Weltmeinung im Zusammenhang mit unseren friedliebenden Schritten.

Die unerquicklichen Ergebnisse des Treffens der Außenminister der vier Mächte in Genf ${ }^{43}$ haben gezeigt, dass die herrschenden Kreise in den Vereinigten Staaten von Amerika, in England und Frankreich bisher nicht den ernstlichen Wunsch haben, sich auf eine völlige Liquidierung der internationalen Spannung einzulassen.

Unsere Aufgabe ist es, unsere Bemühungen in dieser Richtung nicht abzuschwächen, unser sozialistisches Lager ständig zu festigen und beharrlich auf eine Abschwächung der internationalen Spannung hinzuwirken.

Von enormer internationaler Bedeutung war meine Reise mit dem Genossen Bulganin nach Indien, Birma und Afghanistan. ${ }^{44}$ Diese Unterstützung für die Völker, die im Kampf gegen die Kolonialherren stehen, hat große Resonanz und Zustimmung sowohl bei diesen Völkern als auch bei allen fortschrittlichen Kräften gefunden. Diese Reise hat zur Festigung der Positionen des Friedens in den Ländern Asiens und der ganzen Welt beigetragen. Mit aller Klarheit ist jetzt die Schwäche des Einflusses der großen imperialistischen Länder in Ländern wie Indien, Birma und Afghanistan zutage getreten.

In den letzten Jahren, vor allem nachdem das chinesische Volk seine Unabhängigkeit und Freiheit erkämpft hatte, ${ }^{45}$ hat das Streben der Völker der Länder Asiens und Afrikas zur Erringung ihrer Unabhängigkeit und nach Festigung ihrer Beziehungen zur UdSSR und zu allen Ländern des sozialistischen Lagers unermesslich zugenommen.

Das kolonialistische Weltsystem knirscht in allen Fugen. Und das schafft eine neue Lage in der ganzen Welt.

Ich habe das alles aufgezählt, um zu zeigen, welche Maßnahmen uns die Möglichkeit eröffnet haben, unsere Beziehungen zu den kapitalistischen Staaten vom

40 Die sowjetische Führung hatte besonderes Interesse an Indien als maßgebender Macht der Blockfreienbewegung. Der Besuch des indischen Regierungschefs Jawaharlal Nehru in Moskau im Juni 1955 war ein großer Erfolg ihres Bemühens.

41 In der indonesischen Stadt Bandung konstituierte sich vom 18. bis 24. April 1955 die Blockfreienbewegung unter der Führung Indiens, Ägyptens, Indonesiens und Jugoslawiens und akzeptierte das kommunistische China trotz dessen Bündnis mit der UdSSR als Mitglied.

42 Die Konferenz der Staats- und Regierungschefs der drei Westmächte und der UdSSR fand vom 18. bis 23. Juli 1955 statt.

43 Das auf der Gipfelkonferenz im Juli beschlossene Folgetreffen der Außenminister fand vom 27. Oktober bis 4 . November 1955 statt und blieb ohne Ergebnis.

44 Im November und Dezember 1955.

45 Gemeint ist der Sieg der Kommunisten Mao Tse-tungs im Bürgerkrieg mit den Nationalisten Chiang Kai-sheks im Herbst 1949. 
toten Punkt wegzubringen. Doch sie befinden sich trotzdem auf diesem toten Punkt, und das hat eine ziemlich gespannte Lage geschaffen. $\mathrm{Zu}$ den Fragen, die ich genannt habe, sind natürlich auch die Fragen hinzuzählen, deren Regelung wir schon früher erreicht haben. Das sind die Liquidierung des Krieges in Korea $^{46}$ und die Beendigung des Krieges in Indochina. ${ }^{47}$

Ein großer positiver Anfang in der Normalisierung unserer internationalen Beziehungen waren der Abschluss des Friedensvertrags mit Österreich und die Beilegung des Jugoslawien-Konflikts. Die Regelung dieser beiden Fragen, die sozusagen Messlatten unserer Positionen sind, hat eine außerordentlich große Rolle gespielt bei der Normalisierung der internationalen Beziehungen, bei der Abschwächung der internationalen Spannung. Ein weiterer Aufschub des Abschlusses des Friedensvertrages mit Österreich konnte unserer Sache in keiner Weise nützen, weil das durch nichts gerechtfertigt war. Unsere Gegner haben nicht ohne Grund ungefähr so geurteilt: Wenn die Sowjetunion wirklich Frieden will, wozu hält sie ihre gegen den Westen ausgerichteten Truppen in Österreich?

Sie haben darauf spekuliert und die Sowjetunion als Gegner der Unabhängigkeit Österreichs hingestellt. Und sie neigten um so mehr dazu, als es vorher bei der Vorbereitung des Friedensvertrags mit Österreich zwischen der UdSSR, England, Frankreich und den USA fast keinerlei strittigen Fragen gegeben hatte, die einem solchen Vertrag im Wege gestanden hätten.

Daher war es unvernünftig, auch noch weiter auf diesen Positionen in der Österreich-Frage zu bestehen und unsere Truppen zu halten. Solange wir unsere Besatzungstruppen in Österreich belassen haben, haben wir niemanden davon überzeugen können, dass wir keine kriegerischen Absichten hegen. Nach sorgfältiger Abwägung aller Umstände und unter genauer Betrachtung der Österreich-Frage haben wir die Initiative zum Abschluss des Friedensvertrages mit Österreich ergriffen und in dieser Sache große politische Ergebnisse erzielt. Jetzt hat sich Österreich auf den Standpunkt strikter Neutralität ${ }^{48}$ und der Nichtbeteiligung an irgendwelchen Militärbündnissen gestellt. Für uns ist das zweifellos von Vorteil.

Anschließend haben wir alle notwendigen Maßnahmen zur Liquidierung des Konflikts mit Jugoslawien ergriffen. Damit haben wir buchstäblich einen Durchbruch in der Front unserer Gegner erzielt, die ernsthaft damit gerechnet hatten, Jugoslawien in das Lager der aktivsten Länder zu ziehen, die sich in Aggressions-

46 Schon seit dem Frühjahr 1951 war absehbar, dass der am 25. Juni 1950 von Nordkorea begonnene Krieg zu keiner militärischen Entscheidung mehr führen würde. Trotzdem wurden die Kampfhandlungen fortgesetzt, weil Stalin die Truppen der westlichen Beteiligten, vor allem der USA, binden wollte. Erst nach Stalins Tod kam daher Bewegung in die Waffenstillstandsverhandlungen, die dann am 27. Juli 1953 mit einer Vereinbarung zum Abschluss kamen.

47 Nach der Kapitulation der französischen Truppen in der Dschungelfestung Bien Dien Phu vor den Verbänden des kommunistischen Viet Minh kamen die Großmächte zu einer Konferenz in Genf zusammen. In Verhandlungen vom 8. Mai bis 21. Juli 1954 vereinbarten sie einen Waffenstillstand. Vietnam wurde unabhängig und entlang des 17 . Breitengrades in einen kommunistischen Norden und einen nicht-kommunistischen Süden geteilt. Die freien Wahlen, aufgrund deren die beiden getrennten Teile im folgenden Jahr miteinander vereinigt werden sollten, fanden niemals statt.

48 Im Text anschließend durchgestrichen: Es ist kein Werkzeug mehr in den Händen der großen kapitalistischen Staaten. Seine Positionen werden im Rahmen der Neutralität festgelegt. 
blöcken gegen das sozialistische Lager zusammengeschlossen haben. ${ }^{49}$ Wir haben unsere Friedensliebe und den Wunsch bewiesen, uns über jede Frage auf friedlichem Wege zu verständigen, und gezeigt, dass wir tatsächlich den Zustand des Kalten Krieges liquidieren wollen.

Die Liquidierung des jugoslawischen Konflikts, das war wirklich ein Durchbruch [durch die Front] des imperialistischen Lagers, weil seiner Kontrolle ein Land entzogen worden ist, das eine besondere strategische Lage besitzt. Schon eine Neutralisierung Jugoslawiens schafft eine völlig andere Sachlage, ein anderes Kräfteverhältnis im Kampf mit unseren vermutlichen Gegnern. Ich spreche schon gar nicht von der politischen Bedeutung dieser Angelegenheit.

Auf diese Weise hat eine ganze Reihe von Maßnahmen, die in dieser Zeit realisiert wurden, der Sache der weiteren Stärkung unseres sozialistischen Lagers gedient.

Jedoch ist die Gefahr eines neuen Krieges nicht gebannt. Die Imperialisten haben nicht auf ihre Pläne verzichtet, mit Gewalt ihre Ziele zu erreichen. Das müssen wir im Auge haben. Es setzen sich fort das Wettrüsten, die Erweiterung der Aggressionspakte und der Bau von Flottenstützpunkten. Die Westmächte weigern sich, mit der Sowjetunion ein Abkommen zu schließen in der Sache der Schaffung eines Systems der kollektiven Sicherheit in Europa und haben die Regelung dieser Frage von der Regelung der deutschen Frage abhängig gemacht.

Das muss unsere Wachsamkeit gegenüber den Umtrieben der Imperialisten verstärken und nötigt uns dazu, die Macht des sozialistischen Lagers zu stärken und die Anstrengungen aller Länder unseres Lagers besser zu koordinieren.

Die internationale Lage zwingt uns, unsere Kräfte auf dem Gebiet der Außenpolitik weiter zu festigen und die außenpolitischen Verbindungen sowohl des ganzen Lagers insgesamt als auch jedes Landes für sich zu entwickeln. Die Fakten lassen enorme Möglichkeiten in dieser Hinsicht erkennen. Und nutzen wir diese Möglichkeiten? Ich denke, bei weitem noch nicht alle.

Wir haben in dieser Sache starke und schwache Seiten.

Unsere Stärke liegt darin, dass wir in allen internationalen Fragen zumeist in einheitlicher Front aufgetreten sind. Die Sowjetunion und die Länder der Volksdemokratie agieren auf der internationalen Bühne als gewaltige Macht, die alle auf Abschwächung der internationalen Spannung und auf Festigung des Friedens in der ganzen Welt ausgerichteten Maßnahmen unterstützt. Das ist natürlich richtig. Doch wir könnten unsere Tätigkeit elastischer anlegen. Da, wo dies unserem ganzen Lager Vorteil bringt, ist es in einer Reihe von Fällen zweckmäßig, wenn nicht wir, nicht die Sowjetunion, sondern eines der Länder der Volksdemokratie als Initiator in Erscheinung tritt. Die anderen Staaten, darunter auch die Sowjetunion, werden eine derartige Stellungnahme unterstützen. Doch politisch wird es in einer Reihe von Fällen für uns vorteilhafter, eben in dieser Weise vorzugehen.

49 Nachdem die Türkei, Griechenland und Jugoslawien bereits am 18. Februar 1953 in Ankara einen Freundschaftsvertrag geschlossen hatten, wurde am 9. August 1954 zwischen ihnen in Bled zur Abwehr der vonseiten der UdSSR und der mit ihr verbündeten Staaten drohenden Gefahren ein militärisches Bündnis vereinbart („Balkan-Pakt“). 
Ich habe oft mit bürgerlichen Politikern, mit Journalisten zu tun. Sie werfen uns nicht selten vor, dass wir, so heißt es, mit allen Ländern der Volksdemokratie über alle Fragen Vereinbarungen getroffen haben, dass es, so wird gesagt, keinen Fall gibt, in dem irgendein Land aus dem Lager der Länder der Volksdemokratie in dieser oder jener internationalen Frage seine eigene Position bezieht. Davon ausgehend, sagen sie, dass die Länder der Volksdemokratie vorgeblich nicht unabhängig sind, und begreifen nicht, dass die Hauptsache für alle Länder des sozialistischen Lagers die Einheit unserer Politik ist. Die unzertrennliche Einheit unserer Länder ist unsere Stärke, die sich aus der Einheit unserer Klassenstandpunkte ergibt. Bei der Regelung aller prinzipiellen Fragen stehen wir alle auf einheitlichen Klassenstandpunkten und verteidigen dieselben Interessen. Daher gibt es zwischen uns keine Differenzen. Das macht unsere Feinde natürlich fuchsteufelswild. Schließlich wären sie, wenn zwischen unseren Ländern Differenzen entstünden, darüber nur froh. Doch das wäre für unser Lager tragisch, weil eine Spaltung drohen würde, wenn es irgendwelche Abtrünnige gäbe. Differenzen zwischen unseren Ländern würden bedeuten, dass wir keine monolithische Einheit haben. Doch weil keine Differenzen entstehen und auch keine zu erwarten sind, geben wir dem Feind keinen Anlass zur Freude. Unsere Stärke liegt in der Einheit, und diese Einheit müssen wir ständig festigen.

Bei all dem könnten wir unsere Macht [noch] vernünftiger, überlegter einsetzen und unseren Einfluss auf einzelne kapitalistische Staaten sowohl auf der Linie des Einflusses unseres Lagers insgesamt als auch einzeln jedes unserer Länder vergrößern. Ich denke, dass für uns vorteilhaft wäre, dies zu berücksichtigen.

Im Zusammenhang mit den Erfolgen, die wir in der internationalen Arena haben, im Zusammenhang mit der Abschwächung der internationalen Spannung, möchte ich eure Aufmerksamkeit darauf lenken, dass wir jetzt nicht nur eine Abschwächung des Kalten Krieges erreicht haben. Wir führen den Kampf für die Liquidierung des Kalten Krieges, ohne unsere Klassenstandpunkte preiszugeben oder irgendwie von den Prinzipien des Marxismus-Leninismus abzugehen.

Denn wenn wir auf unsere Klassenstandpunkte verzichten, werden die Feinde uns als kluge und weitsichtige Politiker in den Himmel heben. Dann werden auch sie sagen, dass tatsächlich die Zeit zur Liquidierung des Kalten Krieges gekommen ist. Doch darauf werden wir uns niemals einlassen. Auf eine ideologische Abrüstung werden die Feinde bei uns ewig warten müssen. Mehr noch: Dadurch, dass wir unsere Außenpolitik geschickt betreiben und die Lage unseres sozialistischen Lagers und jedes seiner Länder festigen, werden wir unsere Feinde ideologisch entwaffnen, weil immer mehr Menschen in den kapitalistischen Ländern zu begreifen anfangen werden, dass der Kommunismus die Zukunft der ganzen Menschheit ist. Doch solange es zwei Lager gibt: das Lager des Sozialismus und das Lager des Kapitalismus, wird der Kampf zwischen ihnen nicht aufhören.

Wir haben in letzter Zeit große Erfolge erzielt und die Gefahr der Entstehung eines neuen Krieges abgeschwächt. Der Kalte Krieg hat sich, was den Übergang zum heißen Krieg anbelangt, etwas abgeschwächt. Und das wird jetzt von allen eingestanden. Und welchen Lärm die Amerikaner auch machen, sie können die Menschen jetzt nicht vom Gegenteil überzeugen. 
Unser Sieg besteht darin, dass es heute kaum [noch] einer50 glaubt, dass die Sowjetunion und die Länder der Volksdemokratie irgendwelche aggressiven Ziele verfolgen. Diese Legende, auf die sich die Imperialisten so sehr verlassen haben, ${ }^{51}$ genießt kein besonderes Vertrauen mehr. ${ }^{52}$

Früher war das Vorgehen der Imperialisten ganz einfach: Sie haben laut geschrieen, dass die Sowjetunion und die Länder der Volksdemokratie einen Krieg vorbereiten, dass die Russen alles erobern wollen, dass sie bereit sind, die anderen Länder anzugreifen. Und bei diesem Geschrei haben die imperialistischen Monopole die werktätigen Massen noch mehr ausgeraubt, und der Untertan wurde mehr und mehr eingeschüchtert.

Unsere auf Abschwächung der internationalen Spannung gerichteten friedliebenden Maßnahmen, die direkten und klaren Vorschläge der Sowjetunion für ein Verbot der Atom- und Wasserstoffrüstung, für die Reduzierung der konventioneller Rüstungsarten und andere friedliebende Aktivitäten der Sowjetunion und der Länder der Volksdemokratie haben den Leuten den Boden unter den Füßen weggezogen, die ihre Politik auf die lügenhafte Legende von der Aggressivität der Länder des sozialistischen Lagers aufzubauen versucht haben.

Jetzt, als wir mit unseren Stellungnahmen in Indien und Burma und vor allem auf der kürzlichen Sitzung des Obersten Sowjets der UdSSR den Imperialisten in die Fresse gehauen haben, würden sie gerne zur Politik der Einschüchterung der Völker ihrer Länder zurückkehren. Doch man glaubt ihnen nicht mehr. Und dabei ist unser großer Sieg nicht zu übersehen. Wir müssen die Sache in diesem Geist weiterführen.

In letzter Zeit hat die Kommunistische Partei der Sowjetunion eine Politik der Kontaktaufnahme mit der Arbeiterpartei Norwegens unternommen. ${ }^{33}$ Die Norweger haben es abgelehnt, auf eine engere Zusammenarbeit einzugehen, obwohl sie uns nicht absolut zurückgewiesen haben. Sie möchten offenbar die öffentliche Meinung darauf vorbereiten. Doch schon diese Tatsache zeigt, wie weit wir vorangekommen sind in der Sache der Benutzung aller Kräfte, die unsere Position stärken können. Darauf zielen auch unsere Verhandlungen mit den Sozialisten in Frankreich ab, die der Entsendung einer Delegation der Sozialistischen Partei in die Sowjetunion zugestimmt haben. 54 Wir haben vereinbart, dass diese Reise im März stattfinden soll. Auch das zeigt unsere Stärke. Die Sozialisten sind gezwungen, ihre Taktik zu ändern.55 Und das ist eine Bestätigung für die Richtigkeit unserer Politik. Wie Sie schon wissen, hat die Kommunistische Partei Frankreichs vor kurzem bei den Parlamentswahlen einen großen Erfolg errungen, was ein ernsthafter Beweis ist für ihre gefestigte Position, ihre weiter gewachsene

50 Handschriftlich korrigiert statt: niemand [mehr].

51 Handschriftlich durchgestrichen: ist bankrott und.

52 Handschriftlich korrigiert statt: genießt bei den Völkern der kapitalistischen Länder keinerlei Vertrauen mehr.

53 Handschriftlich korrigiert statt: In letzter Zeit haben sich unsere Beziehungen auch mit den sozialistischen Parteien verbessert. Davon zeugt sogar der Kontakt, den die kommunistische Partei der Sowjetunion zur Arbeiterpartei Norwegens aufgenommen hat.

54 Siehe die Dokumente Nr. 7 bis 13 in diesem Band.

55 Handschriftlich gestrichen: und sich auf die Annäherung an uns einzulassen. 
Autorität bei der französischen Arbeiterklasse, bei den werktätigen Bauern und bei der Intelligenz.

Ein weiteres Zeichen für die Stärkung unserer Stellung in der internationalen Arbeiterbewegung sind auch die Verhandlungen mit der Labour Party Englands, die wir über Genossen Duclos 56 führen.

All das zeigt, dass wir es vermocht haben, neue, noch stabilere Positionen zu erobern, unsere Situation als Hort aller progressiven Kräfte zu festigen [und] Bedingungen zu schaffen, die eine Stärkung des internationalen Einflusses unserer kommunistischen Parteien, unseres ganzen sozialistischen Lagers ermöglichen.

Jetzt, Genossen, möchte ich etwas näher auf Fragen eingehen, bei denen wir es für nötig gehalten haben, dass darüber auf so einer Beratung gesprochen wird: die Fragen des weiteren Aufschwungs der Wirtschaft der Länder des sozialistischen Lagers und der Festigung unserer Wirtschaftsbeziehungen. Von der Entscheidung über diese Fragen hängt viel ab.

Offenbar lässt sich keine erschöpfende Regelung für diese Frage formulieren, wir haben zum Beispiel dazu keinen Vorschlag. Doch wir möchten unseren Standpunkt, unsere Ansicht zu den Fragen des Aufschwungs der Wirtschaft unserer Länder zum Ausdruck bringen. Zum Teil ist das [schon] in den Materialien enthalten, die an die Teilnehmer der Beratung verteilt worden sind. Ich denke, wir sollten unsere Ansichten zu diesen Fragen austauschen.

Wir brauchen hier nicht über die Bedeutung der Industrialisierung unserer Länder zu reden. Der Aufbau einer eigenen Schwerindustrie, das ist die Schaffung der notwendigen Bedingungen zur Aufrechterhaltung der Unabhängigkeit für jedes Land, insbesondere für unsere sozialistischen Länder. Die Industrialisierung ist eine Politik, für die unsere Partei gegen viele Feinde gekämpft hat, die sich darum bemüht haben, Kapitalismus in der UdSSR zu restaurieren, unser Land vom Willen der Imperialisten abhängig zu machen. Wir haben stets die Bedeutung der Industrialisierung für die Entwicklung unserer Länder betont. Im Kampf um den Aufbau einer eigenen machtvollen Industrie haben wir gewaltige Siege errungen. Doch es gibt bei uns auch wesentliche Mängel in dieser Sache.

Wenn wir die Tätigkeit der verschiedenen Branchen der Volkswirtschaft analysieren und unsere Erfahrungen bei der Wirtschaftsführung kritisch betrachten, sehen wir, dass bei uns vieles nicht vernünftig genutzt wird. Sie haben die Stellungnahme des Genossen Bulganin auf dem Plenum des Zentralkomitees der KPdSU und andere Materialien des Plenums des ZK der KPdSU gelesen. Wir haben Ihnen das vollständige Stenogramm des Plenums des ZK der KPdSU zugeschickt, und Sie wissen jetzt, welch heftiger Kritik auf diesem Plenum die Mängel unterzogen worden sind, die es bei uns im Bereich der Industrie gibt, welche enormen Möglichkeiten wir für das weitere Wachstum buchstäblich aller Industriezweige haben. Diese Möglichkeiten gibt es nicht nur bei uns, sondern auch in allen Ländern unseres sozialistischen Lagers, des ganzen Lagers insgesamt.

56 Jacques Duclos, Generalsekretär der Französischen Kommunistischen Partei. 
Darum ist eine bessere Nutzung aller uns zur Verfügung stehenden Möglichkeiten bei der Entwicklung der Industrie, beim weiteren noch schnelleren Aufschwung der Wirtschaft unserer Länder eine sehr wichtige Frage.

Auf dem Plenum des ZK der KPdSU, wo die Aufgaben zum weiteren Aufschwung der Industrie der Sowjetunion erörtert wurden, erhielten zum Beispiel große Aufmerksamkeit die Fragen der Spezialisierung der Produktion, der umfassenden Einführung automatischer Produktionslinien in den verschiedenen Industriezweigen, der bestmöglichen Ausnutzung von Werkzeugmaschinen, des Walzmaterials, der Einführung von Druckgussverfahren, des Kampfes um die Reduzierung der Drehbearbeitung und andere Fragen von großer praktischer Bedeutung.

Ich denke, dass solche praktischen Probleme auch in Ihren Ländern auftreten, wenn Sie die Aufgaben bei der Verbesserung der Arbeitsweise der Industrie erörtern.

Doch es gibt nicht nur innere Aufgaben für jedes einzelne Land, sondern auch generelle, für alle unsere Länder geltende Aufgaben bei der Verbesserung der Arbeit der Industrie unseres ganzen sozialistischen Lagers. Mitunter agieren wir da sozusagen separat, jeder für sich. Doch das bedeutet, dass wir unsere Kräfte schwächen.

Wir müssen unsere Möglichkeiten umfassender nutzen. Wir dürfen nicht zulassen, dass jedes Land alles einzeln für sich produziert.

Ich bin kein Referent zu dieser Frage, wir haben ja gar keinen Referenten zu diesem Thema. Doch ich erlaube mir, Genossen, in diesem Zusammenhang einige Worte zu sagen.

Während meines Urlaubs war ich in Rumänien. Einmal bin ich mit dem Genossen Dej ${ }^{57}$ in eine Autofabrik gefahren. Es ist eine gute Fabrik, und man baut gute Autos.

Als die rumänischen Genossen daran gedacht haben, diese Fabrik auf die Produktion von Automobilen umzustellen, haben sie uns um Rat gefragt. Wir haben ihnen eine Reihe unserer Überlegungen mitgeteilt, ihnen geraten, diese Frage sorgfältig zu überlegen und ihnen eine Reihe von Ländern genannt, die keine schlechten Autos herstellen.

Wenn Sie selbst Automobile herstellen, werden das sehr teure Fahrzeuge, das haben wir unseren rumänischen Freunden gesagt.

Und das wissen wir aus eigener Erfahrung. Als wir Automobilfabriken zu bauen anfingen, haben wir Ford um Konsultation gebeten. Ford sagte damals, er könne eine Konsultation geben, wenn die Fabrik nicht weniger als 120000 Fahrzeuge im Jahr herstellt, sind es weniger, steht er als Konsultant nicht zur Verfügung. Die Fabrik wird [dann] nicht rentabel sein. Daher kann er, Ford, sich nicht an so etwas beteiligen. Das war vor drei Jahrzehnten.

Schauen Sie jetzt, was wir machen. Die Rumänen bauen ihre eigenen Autos, die Tschechen bauen ihre, die Deutschen bauen ihre, die Ungarn bauen ihre, die Polen bauen ihre; jeder produziert Autos eigener Marken, und zudem nicht [jeweils] nur ein Automobil [Modell], sondern viele. Nun, Genossen, was ist hier 
vernünftig? Das tun wir, die Marxisten, die das Problem besser begreifen müssten als andere, als beispielsweise die bürgerlichen Ökonomen. Wir verpulvern unsere Kräfte, und folglich schwächen wir unsere Kräfte gegenüber den Kräften des Kapitals. Das ist bedauerlich, aber eine Tatsache.

Deswegen muss man über diese Fragen nachdenken. Können wir uns denn nicht auf eine Zusammenarbeit bei der Produktion von Automobilen einigen. ${ }^{58}$

Oder nehmen wir die Traktorenproduktion. Dieselbe Geschichte passiert auch hier bei der Traktorenproduktion. Und nicht nur bei den Traktoren. Ähnlich sieht die Sache auch in den anderen Bereichen aus.

Ich denke, das ist eine sehr ernste Frage. Hier liegen große Reserven für die Steigerung der Arbeitsproduktivität, für eine bessere Ausnutzung des Kapitalstocks.

Wohin führt eine solche Zersplitterung der Kräfte? Jedes unserer Länder muss große Investitionen für den Bau dieser Fabriken, für die Vergrößerung der Produktionsflächen, den Erwerb der notwendigen Anlagen aufwenden. Würden wir eine Zusammenarbeit durchführen, könnten wir auf den vorhandenen Produktionsflächen zehnmal mehr produzieren. Das ist uns allen wohlbekannt.

Doch sogar wenn wir das begreifen, berücksichtigen wir in der Praxis oft nicht, wohin die Zersplitterung unserer Kräfte führt. Schließlich ist noch vor kurzem Rumänien ein industriell unentwickeltes Land gewesen, dort gab es fast keinen Maschinenbau. Als ich in Rumänien war, sprachen Genosse Dej und ich mit dem Direktor der Automobilfabrik über dieses Thema. Er blickte auf mich wie auf einen Feind, so als wäre ich gekommen, um den rumänischen Genossen die Produktion ihrer Automobile wegzunehmen. Gott mit ihnen, diesen Automobilen, ich habe überhaupt nicht die Absicht gehabt und habe sie auch jetzt nicht, das zu tun! Der Direktor dieser Fabrik ist ein guter Ingenieur, ein hervorragender Direktor, doch er versteht natürlich nicht die ganze Tragweite dieses Problems, er versteht nicht, dass die Zersplitterung unserer Kräfte im Kampf für den Sieg unserer Sache ein allzu kostspieliger Luxus ist.

Wenn wir diese Schwäche nicht überwinden, und das ist nicht unsere Stärke, sondern eine Schwäche, die uns auffrisst, dann werden wir ernstlich darunter leiden.

Bei der Betonung der Notwendigkeit, dass Industriebetriebe sowohl innerhalb unserer Länder als auch zwischen den Ländern unseres Lagers auf breiter Front zusammenarbeiten müssen, möchte ich auch folgende Frage ansprechen.

Nach der bekannten, völlig berechtigten Kritik, die wir zu Hause an der Tendenz zur Unterschätzung der Bedeutung der Schwerindustrie geübt haben, hat unsere Presse einen bestimmten Ton angeschlagen, der auch in den Ländern der Volksdemokratie aufgetreten ist. Es ist sogar die Gefahr entstanden, dass wir in alte Denkmuster in dieser Frage zurückfallen: Wir werden [dann] die Mittel so verteilen, dass wir die in diesen Länder notwendigen Industriezweige benachtei-

58 Es folgen die durchgestrichenen Sätze: Als einziges Land ist Albanien nicht zum Bau eigener Automobile übergegangen, und das wahrscheinlich deswegen, weil es dazu keine Möglichkeiten hat. (Heiterkeit) Wenn sie die Möglichkeiten dazu hätten, würden sie ebenfalls eigene Automobile fertigen. 
ligen, insbesondere die Leichtindustrie und Landwirtschaft. Damit könnten wir uns unglaubliche Schwierigkeiten bereiten.

Wenn man sich die Zahlen genau ansieht in dem Überblick, der vom Genossen Kowal59 verteilt worden ist, dann ist das nach meiner Ansicht schon ziemlich klar zu erkennen. Und das ist eine sehr gefährliche Tendenz.

Wir haben stets den Schwerpunkt auf die Entwicklung der Schwerindustrie gelegt und werden das weiter tun. Doch man muss ein Gefühl für das richtige Maß haben, muss wissen, wie in jeder Etappe der Entwicklung jedes Landes das Verhältnis der Kapitalinvestitionen in der Schwerindustrie, in der Leichtindustrie und in der Landwirtschaft aussehen soll. Sonst, Genossen können wir sehr schwierige Bedingungen schaffen, und in einigen Ländern der Volksdemokratie sind diese schwierigen Bedingungen schon geschaffen worden. Diese muss man liquidieren.

Wir haben nicht genug Fleisch, nicht genug Fett, für die Bevölkerung sind keine ausreichenden Textilien, Schuhe und dergleichen vorhanden, es gibt große Probleme mit dem Wohnraum, wir brauchen Krankenhäuser, und das, Genossen, sind unumgängliche Voraussetzungen für ein normales Leben der Menschen, und deswegen darf man sie [die Voraussetzungen] keinesfalls vergessen.

Jetzt, da die Sowjetunion über eine machtvolle Schwerindustrie verfügt, was von der ganzen kapitalistischen Welt anerkannt wird, da wir eine mächtige Armee mit einer überaus modernen Bewaffnung haben, brauchen wir nicht, wie man so sagt, den letzten Groschen in die Schwerindustrie jedes einzelnen Landes unseres Lagers, sogar beispielsweise Albaniens, zu stecken. Damit werden wir kaum die Verteidigungsfähigkeit eben dieses Albaniens gewährleisten. Die Stärke unserer Länder liegt in ihrer unzertrennlichen Einheit.

Wenn wir die Entwicklung eines jeden Landes unseres Lagers richtig planen und unser Vorgehen ständig koordinieren, haben wir die Möglichkeit, den Aggressor von der Entfesselung eines Krieges abzuhalten und können normalere Bedingungen für die Entwicklung aller Industriezweige, für die Entwicklung der Kultur, Wissenschaft und Kunst sowohl in der Sowjetunion als auch 60 in allen Ländern der Volksdemokratie schaffen.

Man muss anerkennen, dass die Entwicklung der Sowjetunion unter besonderen Bedingungen erfolgt ist. Wir waren der erste Arbeiter- und Bauerstaat, der sich in einem kapitalistischen Umfeld befand. Das alte Russland war ein bäuerliches, agrarisches Land. Der junge Sowjetstaat bedurfte der Entwicklung, während er ringsum von mächtigen Gegnern umgeben war: von leistungsstarken kapitalistischen Industriestaaten. Daher mussten wir all unsere Kräfte darauf ausrichten, unsere Schwerindustrie aufzubauen und alles in die Entwicklung der Schwerindustrie zu stecken, wobei wir [auch] auf das Nötigste verzichtet haben. Wir konnten von niemandem Hilfe erwarten, um uns herum gab es nur Feinde.

Man muss auch berücksichtigen, dass, als wir mit dem Aufbau des Sozialismus begannen, der Lebensstandard in unserem Lande ein ganz anderer war als heute in den Ländern der Volksdemokratie.

59 Konstantin Koval', Vorsitzender des Staatskomitees der UdSSR für Außenwirtschaftsfragen.

60 Handschriftlich gestrichen: noch mehr. 
Heute ist das eine ganz andere Sache. Die Entwicklung der Länder der Volksdemokratie auf dem Weg des Sozialismus vollzieht sich unter den Bedingungen des Bestehens der Sowjetunion. Und das ist eine so große Macht, dass die Feinde mit ihr rechnen müssen. Jetzt muss kein einziges Land unseres Lagers ganz von vorne anfangen, wie wir es zu tun gezwungen waren.

Nehmen wir zum Beispiel die Deutsche Demokratische Republik. Sie ist jetzt unsere offene, entblößte Front im Feuer des Kampfes mit dem Kapitalismus. Dort sind die Grenzen einfach offen, es erfolgt ein ständiger Austausch mit der kapitalistischen Welt in Gestalt der Deutschen Bundesrepublik. Dort tobt nicht nur eine ideologische Schlacht, sondern vor allem eine wirtschaftliche Schlacht zwischen Sozialismus und Kapitalismus. Es findet ein Vergleich statt, welche Struktur die besseren materiellen Bedingungen hervorbringt: die in Westdeutschland oder Ostdeutschland. So lautet heute die Frage.

Nehmen wir zum Beispiel die Tschechoslowakei. Sie ist ein hochentwickeltes Industrieland. Doch bei euch, Genossen Tschechen, gibt es nicht genug Fett und andere Lebensmittel. In Polen und einigen anderen Ländern reichen die Lebensmittel ebenfalls nicht zur Befriedigung der Bedürfnisse der Bevölkerung aus. Wir alle suchen die Produktion der landwirtschaftlichen Erzeugnisse zu steigern. Wir müssen eingehender studieren und rationell verwenden alle unsere Möglichkeiten zur Beschleunigung des Entwicklungstempos der Landwirtschaft. Doch unser Wunsch muss durch reale, vernünftige Kalkulationen in den nächsten Jahren untermauert werden, damit wir ein Programm für alle unsere Länder entwerfen, ermitteln, welche Bedürfnisse sie haben und wie sie befriedigt werden können, in welchen Umfang wir landwirtschaftliche Produkte aus kapitalistischen Ländern importieren werden, vor allem in den ersten Jahren, um mehr Reserven zu haben.

Zur Verbesserung der Landwirtschaft müssen wir den Maschinenbau, die Mineraldüngerproduktion und andere Bereiche entwickeln. Gestützt auf die erzielten Erfolge bei der Entwicklung der Volkswirtschaft müssen wir die Fragen des Aufschwungs der Leichtindustrie und der Landwirtschaft ausarbeiten, um die Erhöhung des Lebensstandards der Werktätigen unserer Länder zu gewährleisten.

Gegenwärtig stellen wir den sechsten Fünfjahrplan zur Entwicklung der Volkswirtschaft der UdSSR auf. In den Ländern der Volksdemokratie werden ebenfalls Pläne ausgearbeitet. Uns allen ist die außerordentliche Bedeutung dieser Arbeit klar. Wir berechnen den Bedarf unserer Länder und stecken die Wege für die weitere Entwicklung ab. Unsere Aufgabe ist es, keine Wiederholung von Fehlern zuzulassen und keine Disproportionen bei der Entwicklung der Wirtschaftszweige zuzulassen. Wir müssen unsere Pläne so erstellen, dass alle materiellen Mittel möglichst richtig und rational verwendet werden. In der Vergangenheit hat es Fehler gegeben, die wir zwischendurch korrigieren mussten. Auch wir waren dabei nicht ohne Schuld, manches haben wir euch „geraten“. Zum Beispiel haben unsere Genossen den rumänischen Genossen geraten, einen Kanal zu bauen, um die Donau mit dem Schwarzen Meer zu verbinden. Diese Arbeit erfordert enorme Kapitalinvestitionen und Arbeitsleistungen. Jetzt ist uns klar, dass dies eine falsche Entscheidung war, weil es in Rumänien wichtigere Probleme gibt. Das Gleiche hat es bei uns gegeben beim Bau des turkmenischen Kanals. 
Wir hatten schon 61 mit dem Bau begonnen, doch dann haben wir überlegt und sind zu dem Schluss gelangt, dass dies jetzt nicht zweckmäßig ist, weil es dringendere und lebenswichtigere Aufgaben gibt. ${ }^{62}$ Lasst uns, Genossen, jetzt nicht solche Sachen ${ }^{63}$ machen.

Ich möchte nochmals auf die Frage der Entwicklung der Deutschen Demokratischen Republik eingehen. Wir wissen alle, welch wichtige politische Bedeutung der Kampf für die Konsolidierung der DDR hat. Die Konsolidierung der DDR, das ist, Genossen, nicht nur eine Sache der Deutschen, sondern von uns allen, von allen Ländern des sozialistischen Lagers. Die Deutschen [in der DDR] können mit dieser Aufgabe nicht allein zurecht kommen, wenn sie den Kampf nur mit ihren Kräften führen. Sie werden deswegen nicht damit zurecht kommen, weil sie es mit einem sehr starken Gegner und einem starken Wirtschaftsgebiet auf seiner Seite zu tun haben. Und ihr Gegner ist auch unser Gegner. Darum müssen wir alle unsere Kräfte mobilisieren und Hilfe leisten zur Konsolidierung der Deutschen Demokratischen Republik. Ich glaube, dass diese Hilfe [bisher] noch nicht ausreicht. Vielleicht ist das scharf formuliert, aber zur Zeit wird die DDR bestohlen. Ich erkläre gleich, worum es dabei geht, Genosse Novotný. ${ }^{64}$ Wenn in der Deutschen Demokratischen Republik eine neue Maschine oder ein gutes Gerät produziert wird, dann wollen wir wie die Kapitalisten [lediglich] ein Exemplar kaufen, suchen wir das Herstellungsverfahren zu ergründen oder tüfteln selbst etwas aus und beginnen es in unserem Land zu produzieren. Auf diese Weise werden die Möglichkeiten der Deutschen Demokratischen Republik bei der Produktion einer Reihe von Maschinen und Geräten beschränkt. Folglich verringert das ihre Möglichkeiten. Die DDR sollte für die von ihr hergestellten Maschinen die Waren erhalten, die sie benötigt. Man kann noch weitere derartige Beispiele dieser Art anführen. An dieser Sache tragen nicht nur wir, die Vertreter der Sowjetunion, Schuld, sondern auch die der anderen Länder der Volksdemokratie. Wenn wir so vorgehen, schwächen wir unsere Freunde, die ihre Errungenschaften gerne [mit uns] teilen. Wir sollten auch künftig unsere Erfahrungen untereinander austauschen. Doch dabei, Genossen, müssen die Fragen richtig geregelt werden, sodass es keine Beeinträchtigung der Interessen irgendeiner Republik gibt.

Oder nehmen wir folgendes Beispiel. Wir plagen uns damit, neue Fabriken zu bauen, während die Deutsche Demokratische Republik in ihren Industrieunternehmen unausgelastete Kapazitäten hat. Ist das denn vernünftig? Wieso nutzen wir diese Möglichkeiten nicht? Vielleicht werden dies manche mit der, wie es dann heißt, Angst vor einer Entwicklung der Industrie der DDR zu erklären versuchen, weil diese Industrie unweit der westlichen Länder gelegen ist. Wie denn, Genossen, wollen wir etwa vor dem Westen zurückweichen? Ich denke, dass solche Überlegungen grundfalsch sind.

61 Das Wort „schon“ wurde handschriftlich eingefügt.

62 So die handschriftliche Korrektur, die folgenden Text ersetzt: aber dann hat man gefragt: Wem nützt das? Man hat überlegt und ist zu dem Schluss gelangt, dass dies zu tun unzweckmäßig ist.

63 Handschriftlich korrigiert statt: keine Dummheiten.

64 Antonín Novotný, Erster Sekretär der Kommunistischen Partei der Tschechoslowakei. 
Wir müssen die Produktionsmöglichkeiten, die technischen Kader der DDR, die deutsche Produktionskultur intelligent nutzen, was den deutschen Arbeitern und vor allem der technischen Intelligenz Zuversicht gibt. All das wird unsere Positionen und Möglichkeiten im Kampf mit den Imperialisten des Westens festigen und verstärken.

Das Gleiche kann man auch zur Tschechoslowakei sagen und nicht nur zur Tschechoslowakei. Zum Beispiel produziert die Sowjetunion das Flugzeug IL-14, doch auch die Tschechen und Polen bauen es. ${ }^{65}$ Dabei wird nicht berücksichtigt, dass wir gar nicht so viele Flugzeuge vom Typ IL-14 brauchen. Doch wir bauen sie in mehreren Ländern. ${ }^{66}$ Das ist doch eine direkte Verschwendung menschlicher Energie und materieller Ressourcen. Und im Ergebnis führt dies zu einer Verminderung unserer potentiellen Möglichkeiten bei der Entwicklung der Wirtschaft.

Auch bei der Produktion der Militärtechnik sündigen wir. Es genügt, beispielsweise darauf hinzuweisen, dass zum Beispiel die Polen und Tschechen Flugzeuge produzieren, deren Produktion in der UdSSR eingestellt wurde. ${ }^{67}$ Es ist zum Beispiel kaum nötig, die Produktion des Flugzeugs IL-15 zu fördern, das schon lange keinen Wert mehr hat. Vernüntig bei dieser Organisation der Produktion der Militärtechnik ist [nur] wenig. So sind die Dinge nicht nur beim Flugzeugbau, sondern auch bei der Artillerie und bei einer Reihe anderer Bereiche des Wehrwesens. Die Technik, welche die Tschechen und Polen produzieren, entspricht nicht dem heutigen Stand. Schließlich haben wir nicht vor, [schon] morgen Krieg zu führen. Folglich haben wir Zeit. Doch einige Geräte, die jetzt produziert werden, so lässt sich im Voraus sagen, werden nicht für den Kampf geeignet sein. Daher müssen unsere Militärs und die Leiter der entsprechenden Behörden unsere Produktion kritisch überprüfen. Zwar glauben die Militärs: je mehr Technik, desto besser. Doch wir müssen ihnen die richtige Richtung vorgeben. Wir müssen diese Fragen erörtern, über all das nachdenken und richtig entscheiden.

Ich möchte einige unserer Überlegungen zur Entwicklung der Militärtechnik vortragen. Im Fünfjahrplan der UdSSR legen wir das Hauptaugenmerk im Bereich der Verteidigung auf die Entwicklung der Luftwaffe, weil die Luftwaffe mit der Entwicklung der Atom- und Wasserstoffrüstung sehr große Bedeutung erhält. Die Kernwaffe ist eine Waffe, die auf das Bewusstsein einwirkt und das Bewusstsein vieler Imperialisten ernüchtert, diese Waffe wird dabei helfen, den Krieg fernzuhalten. Das heißt natürlich nicht, dass wir die Produktion der anderen Waffen völlig fallen lassen. Wir tun alle Erforderliche, doch in gewissen Proportionen. Die Hauptsache ist die Luftwaffe für den Nah- wie für den Fernkampf.

Die Luftwaffe für den Nahkampf befindet sich bei uns auf hinreichend hohem Stand, und wir produzieren sie in ausreichender Stückzahl. Wir ergreifen Maßnahmen zur weiteren Entwicklung der Luftwaffe für den Krieg über große Ent-

65 Handschriftlich durchgestrichen: und jetzt denken auch die Albaner daran, es zu produzieren.

66 Handschriftlich durchgestrichen: Wie viel kostet dieses Flugzeug?

67 Handschriftlich durchgestrichen: Dazu ist zu sagen, dass vielleicht ein Leck besteht usw. 
fernungen hinweg. Dabei ist zu beachten, dass die Raketentechnik mit Wasserstoffsprengköpfen die Fragen der Kriegführung auf andere Weise löst.

Wir müssen, Genossen, unsere Stärke und unsere Möglichkeiten, die Aggressoren zu zügeln, vernünftig nutzen, um keinen Krieg zuzulassen. Wir müssen Beharrlichkeit und Standhaftigkeit zeigen und dürfen uns nicht einschüchtern lassen vom Kriegsgeschrei, bei dem die Imperialisten wahre Meister sind. Dulles ${ }^{68}$ nimmt Stellung, noch einer, ein dritter, zehnter, sie wollen uns einschüchtern, damit wir zur Seite weichen.

Doch wir haben starke Nerven, und vor allem verfügen wir über die Macht, den Aggressoren Zügel anzulegen. Ich glaube, dass sich die internationale Lage unserer Länder wesentlich gebessert hat. Wir haben feste Positionen erkämpft. Die letzte Explosion unserer Wasserstoffbombe hat bei den Imperialisten einen besonders starken Eindruck hinterlassen. Folglich haben sich die Ausgaben für die Produktion der Wasserstoffbombe gelohnt, weil diese Waffe die Aggressoren abschreckt. Wir bereiten noch eine Bombe vor, um unsere Gegner weiter zu ernüchtern, um sie am Bramarbasieren zu hindern. Unsere Stärke ist jetzt kolossal, sowohl die militärische als auch die moralisch-politische. Wir müssen diese Stärke im Interesse unserer Völker, im Interesse des sozialistischen Lagers vernünftig nutzen.

Jetzt, Genossen, möchte ich noch eine Frage beleuchten. Gestern haben wir auf der Sitzung des Präsidiums des ZK der KPdSU folgenden Beschluss gefasst im Zusammenhang mit der Ausarbeitung des sechsten Fünfjahrplans der UdSSR: in diesem Fünfjahrplan zum 7-Stunden-Tag überzugehen. Den Übergang ab dem Jahr 1957 beginnen und ihn am Ende des Fünfjahrplans abzuschließen. In diesem Jahr, nach dem XX. Parteitag wird der Übergang zum 6-stündigen Arbeitstag am Samstag und vor Feiertagen durchgeführt.

Wir glauben, dass wir Ihnen das sagen sollten, weil wir dabei im Auge haben, dass diese Frage nicht nur innenpolitische Bedeutung für die UdSSR, sondern auch große internationale Bedeutung hat. In dieser Frage machen wir Ihnen keinerlei Vorschläge, sondern informieren Sie nur über diesen Beschluss. Diese Frage bereiten wir für den Parteitag vor, vorerst werden wir dies nicht veröffentlichen und haben es nur Ihnen gesagt.

Unsere Aufgabe besteht darin, sowohl in internationalen als auch in innenpolitischen Fragen keine Möglichkeit auszulassen zur Stärkung unserer Wirtschaft, unseres sozialistischen Lagers.

Ich möchte noch einige Worte sagen zu meiner Reise mit Genossen Bulganin nach Indien, Birma und Afghanistan. Diese Reise war ein großes politisches Ereignis, hat eine große Rolle gespielt bei der weiteren Verbesserung unserer Beziehungen zu diesen Ländern und bei der Festigung der Sache des Friedens. Unsere Reise hat noch mehr die Schwäche der Position der imperialistischen Staaten in Indien, Birma und Afghanistan hervortreten lassen, sie hat das grundlegend veränderte Kräfteverhältnis zwischen Kapitalismus und Sozialismus enthüllt. In unseren Stellungnahmen haben wir den Kolonialismus entlarvt. Das hat natürlich Erbitterung im imperialistischen Lager hervorgerufen. Die bürgerlichen Journa-

68 John Foster Dulles, amerikanischer Außenminister. 
listen haben geschrieben, dass unser Verhalten aggressiv ist. Ja, es war wirklich aggressiv gegenüber den Imperialisten, den Kolonialherren. Wir sind der Ansicht, dass so eine Haltung absolut richtig ist.

Während dieser Reise haben Millionenmassen den Hass der Völker gegen die Imperialisten und ihre gewaltige Liebe und ihr Vertrauen zur Sowjetunion und zu den Ländern des sozialistischen Lagers gezeigt. Wir haben auch vorher schon gewusst, dass die Werktätigen dieser Ländern Liebe zur Sowjetunion und zu den anderen Ländern des sozialistischen Lagers hegen, doch was wir dort gesehen haben, übertraf alle unsere Erwartungen und Vorstellungen. Dort begrüßte man nicht Chruschtschow, nicht Bulganin, man begrüßte unser Volk, man begrüßte die sozialistische Ordnung, man begrüßte die marxistisch-leninistische Lehre.

Man sagt, dass nach uns irgendeiner von den Politikern der USA in diese Länder reisen will. In der Presse heißt es, dass Dulles eine Reise dorthin plant. Doch wozu will er dorthin?

Unsere Reise hat ebenfalls gezeigt, dass wir unsere Möglichkeiten zur Entwicklung der Beziehungen zu den Ländern Asiens nicht genug nutzen. Wir haben das auf der Reise gespürt und gesehen, vor allem in Indien und Burma. In Afghanistan herrschen andere Bedingungen. Die Bewegung der Völker Asiens im Kampf für ihre Unabhängigkeit, das ist eine kolossale Macht im Kampf gegen die imperialistischen Staaten. Aufgrund des nationalen Befreiungskampfes kommt es zum Zerfall des Kolonialsystems. Und wie sich Dulles und andere Drahtzieher des Imperialismus auch immer bemühen werden, es wird ihnen nicht gelingen, diesen historischen Prozess zu stoppen.

Die Reise der Vertreter der Sowjetunion in die Länder Asiens ist hoch bedeutsam. Sie wird lange diskutiert werden und ihren Nachhall finden; sie wird sich nicht nur auf Asien, sondern auch auf Afrika und andere Regionen und Kontinente auswirken.

Jetzt möchte ich sagen, wieso wir uns auf der Sitzung des Obersten Sowjets der UdSSR und auf der Pressekonferenz in Indien so genau und recht scharf über das Kominform ${ }^{69}$ geäußert haben.

Auf einem der Treffen mit Nehru hat er uns gesagt, dass er mit uns sprechen möchte und dass an diesem Gespräch nur ich und Gen. Bulganin uns beteiligen sollen. Nehru sagte, dass er nur den Dolmetscher mitbringt.

Wir haben uns mit ihm getroffen. Dabei sprach er die Kominform-Frage an. Nehru sagte: Wozu ist so eine Organisation nötig [und meinte], dass das Kominform eine Einmischung in die inneren Angelegenheiten anderer Staaten sei. Er sagte es nicht direkt, doch das war der Sinn. Er fing damit an, dass die indische kommunistische Partei Mittel erhält, viel Eigentum erwirbt, doch die Quellen dieser Mittel sind nicht bekannt.

Er sagte weiter, dass es eine Zeitung gibt „Für dauerhaften Frieden, für Volksdemokratie!“ - das [vom Kominform herausgegebene] führende Organ der kom-

69 Das Kommunistische Informationsbüro (Kominform) war im September 1947 von Stalin als Kampforganisation gegen den Westen gegründet worden, hatte aber seine Bedeutung weitgehend verloren, nachdem sich der Generalsekretär der Italienischen Kommunistischen Partei 1950/51 geweigert hatte, nach Moskau zu kommen und dort die Leitung zu übernehmen. 
munistischen Parteien. Alle Artikel in dieser Zeitung der kommunistischen Parteien werden als Direktiven, als verbindlich für die Führung betrachtet. Vor diese Fragen hat uns Nehru gestellt.

Wir haben ihm so geantwortet, wie wir es später auf der Pressekonferenz und auf der Sitzung des Obersten Sowjets der UdSSR zum Ausdruck gebracht haben. Wir haben gesagt, dass es keinerlei Grundlage dafür gibt, diese Fragen zu stellen. Wieso können die Sozialisten internationale Organisationen haben, und das ruft bei niemandem Einwände hervor. Wieso können die Kapitalisten ihre Organisationen haben? Wir haben darauf hingewiesen, dass es nicht richtig ist, die Kominform-Frage zu stellen, dass dies eine Einmischung in die Angelegenheiten der kommunistischen und Arbeiterparteien ist, dass dies eine Frage unserer Parteien ist und dass sie diese so entscheiden, wie sie es in ihrem Interesse, im Interesse der Völker unserer Länder, im Interesse der proletarischen Solidarität für richtig halten. Unsere Parteien brauchen auch eine Zeitung, und das ist Sache unserer Parteien.

Nehru hat gesagt, dass ihm das alles klar ist, doch er spricht davon, damit wir es wissen. Nach diesem Gespräch mit Nehru haben wir beschlossen, uns zu dieser Frage auf der Pressekonferenz und danach auf der Sitzung des Obersten Sowjets zu äußern.

Wieso musste diese Frage so scharf gestellt werden? Deswegen, weil diese Frage gewaltige prinzipielle Bedeutung hat. Wir reden von der Liquidierung des Kalten Krieges, von der Abschwächung der internationalen Spannung. Die Bourgeoisie versucht das so zu darzustellen, dass wir angeblich auf die marxistische Lehre verzichten, dass wir Positionen aufgeben. Deswegen greifen sie solche Fragen auf wie die Kominform-Frage usw.

Eben deswegen mussten wir darüber reden, dass wir für die friedliche Koexistenz sind, und das bedeutet die Anerkennung der Existenz von zwei Systemen. Hier ist die sozialistische Ordnung und dort die kapitalistische, das ist unsere Ideologie, und so sieht das Wesen der Ideologie des Kapitalismus aus. Wir sagen dem Imperialisten direkt: Ihr könnt uns und unsere sozialistische Ordnung nicht liquidieren, und wir können euch nicht liquidieren, wir haben nicht vor, unsere Struktur des Sozialismus irgendwohin zu exportieren. Die Völker selbst eines jeden Landes müssen die für sie geeigneten Verhältnisse wählen. Doch solange sowohl das sozialistische als auch das kapitalistische System bestehen, müssen sie irgendwie miteinander auskommen, weil wir alle auf einem [einzigen] Planeten leben. Wir müssen friedlich koexistieren. Das haben wir recht deutlich gesagt und sagen es weiterhin.

Die Frage des Informationsbüros der kommunistischen und Arbeiterparteien hat große grundsätzliche Bedeutung für alle kommunistischen Parteien, darunter auch für die Kommunistische Partei der Sowjetunion. Das ist wichtig für die Erziehung der Arbeiterklasse. Man muss wissen, wo die Grenzen der friedlichen Koexistenz verlaufen, um unsere Politik richtig in die Praxis umzusetzen.

Ich denke, dass wir richtig gehandelt haben, als wir diese Erklärungen zum Kominform abgegeben haben. Wo es um prinzipielle Fragen, um unsere Ideologie geht, dürfen wir nie ein Zugeständnis machen, wenn das den Verzicht auf unsere marxistisch-leninistischen Grundsätze bedeutet. Sollen sich doch die Im- 
perialisten an die bestehende Lage gewöhnen, sollen sie doch wissen, dass sie von uns eine ideologische Abrüstung niemals erwarten können.

Das sind die Fragen, die ich bei der Eröffnung unserer Beratung für notwendig erachtet habe.

Die Tagesordnung ist an alle verteilt worden. Es ist kaum erforderlich, sie vorzulesen. Es wird der Vorschlag eingebracht, alle zwei Stunden eine Pause von 20 Minuten einzulegen. Den Vorsitz der Beratung sollten die Leiter der Delegationen der Reihe nach übernehmen. Alle zwei Stunden, d. h. nach jeder Pause, ein neuer Vorsitzender.

Wenn es keine Einwände gibt, dann wird auf der heutigen ordentlichen Sitzung der Vertreter der UdSSR den Vorsitz übernehmen, auf der nächsten der Vertreter Albaniens und danach gemäß dem Alphabet.

Es gibt keine Einwände? Nein.

Wir sind der Ansicht, dass ein Sekretariat bestehend aus einem Vertreter pro Delegation eingerichtet werden sollte. Aus unserer Delegation benennen wir Gen. Ponomarjow. ${ }^{70}$ Wir bitten, aus jeder Delegation einen Vertreter zu benennen.

Übersetzung aus dem russischen Originaltext

RGANI, fond 52, opis' 1 , delo 443, Bl. 1-30

\section{Gespräch mit dem Führungsmitglied der britischen Labour Party Harold Wilson am 12. Januar 1956 (Auszug)}

Nach einer Unterhaltung über weniger wichtige Fragen, zuletzt über Vorgänge vor dem Zweiten Weltkrieg, wendet sich Chruschtschow im Gespräch mit Wilson ${ }^{71}$ aktuellen Kernproblemen des Ost-West-Verhältnisses zu.

Chruschtschow sagt, dass er befürchtet, die Geschichte könnte sich jetzt in gewissem Umfang wiederholen, auch wenn sich die Rolle der Staaten verändert hat. Die unschöne Rolle des Anführers im Kampf gegen die Sowjetunion haben jetzt die USA übernommen, wo es nicht wenige Leute gibt, die glauben, man könne Ideen mit Hilfe von Kanonen bekämpfen. Davon ausgehend, dass die USA weit von der UdSSR entfernt sind, möchten sie einen Krieg auf dem europäischen Kontinent anzetteln, um danach ihren Willen zu diktieren.

Man merkt, fährt Chruschtschow fort, dass England dazu eine etwas andere Einstellung hat, dass es gegenüber einer solchen Politik einen gewissen Widerstand zeigt, obwohl es in einiger Hinsicht diesen Kurs auch selbst unterstützt.

70 Boris Ponomarëv, für die Beziehungen zu den anderen kommunistischen Parteien zuständiger Sekretär des ZK der KPdSU.

71 Harold Wilson (1916-1995) wurde 1945 als Labour-Abgeordneter ins Unterhaus gewählt und als Minister in das bis 1951 regierende Kabinett von Clement Attlee berufen. Als die Labour Party ab 1951 in der Opposition war, übernahm er die Rolle des Schatzkanzlers (Finanzministers) in ihrem Schattenkabinett, an dessen Spitze seit 1955 Hugh Gaitskell stand. 